\title{
Correspondence
}

\section{An acute ICU in a Maximum Secure Hospital}

Sir: September 1994 saw the opening of an acute Intensive Care Unit (ICU) at Ashworth Hospital one of three Special Hospitals catering for dangerous mentally disordered patients. It was envisaged that such a facility would prevent behaviourally disturbed individuals being transferred at times of crises to high dependency wards to the detriment of the resident chronically mentally ill patients.

Initial demand suggests that the ten bedded unit is too large for the hospital population it serves, with only five or six beds utilised at any one time. Part of the reason for this underutilisation is the culture within the special hospital: staff feel they have failed if they find themselves unable to manage a patient, however disturbed he is.

Proposals are now being considered to offer this service to possible purchasers such as prisons, district general hospitals and regional secure units (RSUs). Negotiations are ongoing between hospital managers and the Home Office regarding conditions for possible short-term transfer of restricted patients. Transfers from other health service institutions will only take place on Section 17 leave of absence conditions. This will facilitate speedy return of patients once recovered and prevent the ICU silting up or being used as a back door to the Special Hospital, bypassing its strict admission criteria. To date two such transfers have taken place, one patient from an RSU and another from a high dependency ward of a local psychiatric hospital. It is likely that transfers from prisons would present particular problems as urgent assessment would be required to determine the prisoner's detainability under the Act and prevent inappropriate transfer of violent. non-mentally disordered prisoners.

Other proposals totally contrary to the concept of an active ICU have been suggested on grounds of financial viability, e.g. the ICU be used to manage chronically dangerous patients who require frequent periods of seclusion and also be utilised for the management of patients with chronic challenging behaviours. The possibility exists therefore that the ICU may gradually evolve into a ward for chronically disturbed patients which will invariably be regarded both within and without the hospital as a refractory ward.

Rosemary Brook and Placid R. COOREY Ashworth Hospital, Liverpool L31 1HW

\section{The College Library}

Sir: Reading the article on the centenary of the College Library (Psychiatric Bulletin. September 1995, 19, 577-579) made me realise how far the present library of the College has progressed in 100 years. We are now reaching the end of the millennium, and I would like to suggest some ways through which the College Library and Information Service can be brought into the 21 st Century.

My increasingly large and unwieldy collection of Bulletins and Journals is almost wasted space as far as ease of access to information is concerned. It is not beyond the College to allocate resources for the compilation of a computerised index for both these publications. The prospect of effortless searches of subjects, by authors or article title is worth the effort. It is now also possible to use 'document capture' software and a scanner to have the whole article in a computerised form. Long runs of the Psychiatric Bulletin and British Journal of Psychiatry can be published on CDROM. It would be expensive to produce but should sell well to anyone with libraries and people with shrinking shelf-space.

A more ambitious project would be to have online facilities installed in the library and the College allowing electronic mail, remote searches of the catalogue, and access to specialised literature searches. Another benefit of connecting the College Library to a network is the possibility of establishing our own presence on the Internet. Having our 'site' would allow information about the College, its documents, policy statements and campaigns like 'Defeat Depression' to reach out worldwide to many millions. How about having these as the College's own millennium project?

\section{Aguilina}

Department of Psychiatry, Royal Free Hospital, London NW3 2QG

Sir: Dr Aquilina's letter is timely. The College Information Technology Working Party that I chair is looking at the needs of the College administratively, the Membership and the general public. This includes College networking. internal and external e-mail and a College site on the World Wide Web. The Committee has already made some interim proposals and will report fully within the next six months.

IAN PULEN

The Royal College of Psychiatrists, London 